\title{
Therapeutic Efficacy of Antibacterial Ocellatin Peptides- A Comprehensive Review
}

\author{
Chandra Sekar Ponnusamy ${ }^{1(\mathbb{D})}$, Rajasekaran Ramalingam ${ }^{1, *(\mathbb{D})}$ \\ 1 Bioinformatics Lab, Department of Biotechnology, School of BioSciences and Technology, VIT Deemed to be University, \\ Vellore, Tamil Nadu, India \\ * Correspondence: rrajasekaran@vit.ac.in (R.R.);
}

Scopus Author ID 21739948000

Received: 22.09.2021; Revised: 20.10.2021; Accepted: 24.10.2021; Published: 20.11.2021

\begin{abstract}
Antimicrobial peptides (AMPs), ascribed to their decreased microbial drug resistance, can be employed as potent small-molecule drugs to treat various diseases. AMPs have been conserved in a wide variety of living organisms as a result of the evolution of the innate immune system. Notably, Ocellatin AMPs derived from South American Leptodactylus genus frogs have a higher therapeutic efficacy against infections. Inhibitory activity of Ocellatin AMPs against bacterial membranes is determined by the dynamic interplay of peptide cationic, hydrophobicity, helicity, and amphipathicity. Another advantage of using AMPs as drug candidates is their cell selectivity that is non-hemolytic to human cells. Ocellatin AMPs with optimal hydrophobic residues would therefore be a recommended therapeutic candidate. Henceforth, such AMPs could be used as an alternative strategy in curbing antimicrobial resistance. It is noteworthy that the therapeutic efficacy of Ocellatins is to be appreciated for its broad application as it has been proved to be active against several humans, animal, and plant bacterial pathogens.
\end{abstract}

Keywords: antimicrobial peptides; Ocellatin, antibiotic resistance; membranolytic peptides; therapeutic modifications.

(C) 2021 by the authors. This article is an open-access article distributed under the terms and conditions of the Creative Commons Attribution (CC BY) license (https://creativecommons.org/licenses/by/4.0/).

\section{Introduction}

Antibiotics, invented by Alexander Fleming after World War I, have been used as a wonder drug for almost a century [1]. However, such antibiotics are becoming a failure due to their extensive overuse in recent decades, resulting in antimicrobial resistance (AMR) [2]. This alarming rate of antibiotic failure prompted scientists to emphasize alternate therapeutics [35]. Nothing in biology makes sense unless viewed from the perspective of evolution, from which we seek asylum in antimicrobial peptides (AMPs), that has evolved as innate host molecules in a wide range of organisms around 2.5 billion years ago [6-8].

Compared to AMPs from other organisms, AMPs isolated from the skin secretion of anurans (frogs and toads) hold out as a promising therapeutic candidate for antimicrobial resistance [9]. Generally, the skin serves as a gateway between an organism's internal and external environments, as well as a barrier to a variety of microbes attempting to invade our cells as their home [10]. The skin of anurans, in particular, has evolved AMPs to enable them to survive and thrive in both terrestrial and aquatic habitats [11]. According to Antimicrobial Peptide Database-3 (APD3), over 980 AMPs out of 1093 anuran AMPs demonstrated antibacterial activity [12]. 
More specifically, experimental studies have established that Ocellatin peptides derived from the skin secretions of Leptodactylus genus frogs have a broad spectrum of antibacterial activities [13]. Nascimento et al., 2004 first discovered Ocellatin-1 in the skin dorsal glands of the Amazonian Argus frog, Leptodactylus ocellatus, and reported it to be active against the Gram-negative bacterium E.coli. Inconsequent studies, the peptides from the same species [1416] and evolutionarily similar frog species $L$. pustulatus [17], L. syphax [18] L. validus [19] $L$. laticeps [20], and L. labyrinthicus [21,22] have revealed the membranolytic activities against several bacteria including multidrug-resistant strains [23]. Moreover, recent studies on $L$. latrans [24], L. vastus [25], L. insularum [26], and L. nesiotus [26] have further revealed antibacterial properties of newly characterized Ocellatin AMPs. Considering the lack of inclusive data regarding this emerging topic, a comprehensive review of the aforementioned Ocellatin peptides in terms of their therapeutic potential is presented.

\section{Basic Features of Anuran AMPs and their Action Mechanism}

Anuran AMPs are mostly alpha-helical amphipathic peptides containing hydrophobic and hydrophilic residues with a range in length from 5 to 50 amino acids. In general, these AMPs are cationic because they contain more positively charged amino acids such as lysine $(\mathrm{K})$, arginine $(\mathrm{R})$, and histidine $(\mathrm{H})[27,28]$. The cationic property, frog-skin peptides in their early phase are electrostatically attracted to the anionic bacterial membrane [29]. Following that, such AMPs self-associate as carpet, barrel-stave, or toroid models, as illustrated in Figure 1.

Self-associated AMPs can permeabilize bacterial membranes in two ways either micellization or pore formation [30]. For instance, Aurein AMP from the Golden-bell frog (Litoria aurea) develops a detergent-like carpet model after reaching a particular threshold level and aggregates around the membrane, leading to micellization [31]. On the other hand, toroidal and barrel-stave models result in pore formation, leading to membrane depolarization and cell death. For instance, the Magainin AMPs from African clawed frog (Xenopus laevis) assemble into transmembrane pores of the toroidal type [32]. Also, the buforin AMPs from Asiatic toads (Bufo gargarizans) self-associate in the barrel-stave paradigm, which allows hydrophobic components of peptides to interact with hydrophilic membrane surface [33]. As a result, a transmembrane pore forms with a core lumen, which is followed by an efflux of cellular constituents and consequent cell death.

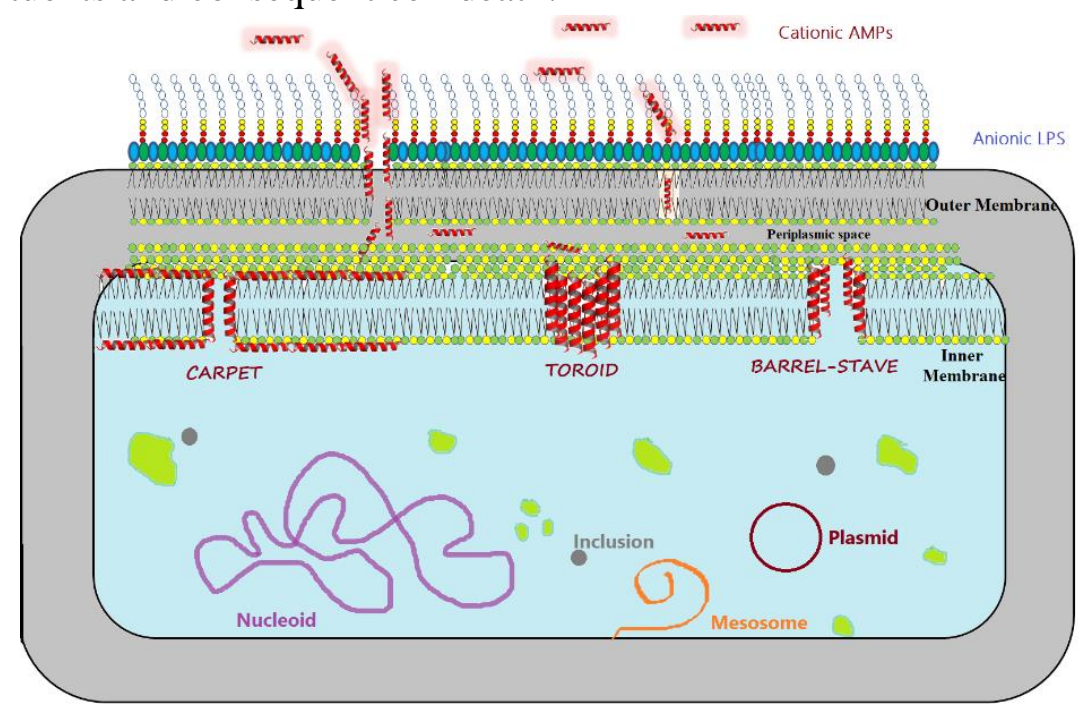

Figure 1. General mechanism of action of anuran alpha-helical AMPs against the bacterial membrane. 


\section{Selective Permeability of Ocellatin Peptides}

In general, AMPs with high bacterial permeability are considered valuable in therapeutics, where the cell permeability is determined by amphipathic (hydrophobic and hydrophilic) residues [34]. Amphipathicity of AMPs ought to be optimal because a greater value will enable them to penetrate eukaryotic membranes, while a lower value will prevent an AMP from even penetrating bacterial membranes [35,36]. However, Ocellatin AMPs with optimum hydrophobic residues would indeed be a preferred therapeutic priority, which has been demonstrated in various investigations. Moreover, the self-associating tendency of AMPs is also dependent on their immense amphipathic values [37]. Similarly, increased amphipathic values found in Ocellatin-4 exhibit a cascade of antibacterial activity as a result of its selfassociation in solutions.

Another property of AMPs that determines membrane permeability is their cationic [38]. In an experimental evaluation of Ocellatin-L2, it has been revealed that when negatively charged residues in the peptide were replaced with neutral residues, the peptide's capacity to inhibit E.coli was compromised. Positive charges in peptides have been proven to exhibit enhanced bacterial permeability [39]. On the other hand, cationic Ocellatin AMPs selectively avoid adhering to zwitterionic eukaryotic membranes. AMPs are notably specific towards bacterial cells due to their net charge. For instance, in Ocellatin-4, two lysine residues have been neutralized by two aspartate residues, enabling it to be both antibacterial and nonhemolytic [40]. Conversely, the presence of an extra negatively charged aspartate residue in Ocellatin-PT peptides impedes negatively charged bacterial membrane, resulting in its decreased antibacterial efficacy. In our previous computational analyses, we revealed that Ocellatin-1's net charge was lower than Ocellatin-F1, Ocellatin-K1, and Ocellatin-S1 due to the presence of negatively charged glutamic acid at position 23 (E23) [40]. Moreover, this conservation of E23 is found consistent with experimental results on Ocellatin-V1, OcellatinV2, Ocellatin-V3, and Ocellatin-P1.

\section{Antibacterial Efficacy and Biofilm Inhibiting Potency}

It is worth noting that numerous Ocellatin AMPs are efficient against a wide variety of bacterium, as denoted in Figure 2 [20]. Several Gram-negative bacteria, including Escherichia coli (E.coli), Klebsiella pneumoniae (K. pneumoniae), and Salmonella choleraesuis (S. chloeraesuis) were inhibited by Ocellatin-PT1, Ocellatin-PT2, Ocellatin-PT4 and OcellatinPT8 peptides produced from L. pustulatus [24]. Peptides such as Ocellatin-S1 are equally effective to Gram-negative bacteria such as Escherichia coli and Gram-positive bacteria such as Staphylococcus aureus (S. aureus). However, various peptides, including Ocellatin-P1, Ocellatin-L1, and Ocellatin-F1, have been found to be more efficient against Gram-negative than Gram-positive bacteria (Table 1).

\begin{tabular}{|c|c|c|c|c|c|c|}
\hline S.No & $\begin{array}{c}\text { Peptide } \\
\text { name }\end{array}$ & $\begin{array}{l}\text { UNIPROT } \\
\text { ID }\end{array}$ & Species & Sequence & Active against & Reference \\
\hline 1 & Ocellatin-1 & P83951 & L. ocellatus & GVVDILKGAGKDLLAHLVGKISEKV & E.coli & [14] \\
\hline 2 & Ocellatin-2 & P83866 & L. ocellatus & GVLDIFKDAAKQILAHAAEQI & E.coli & [14] \\
\hline 3 & Ocellatin-3 & P83867 & L. ocellatus & GVLDILKNAAKNILAHAAEQI & E.coli & [14] \\
\hline 4 & Ocellatin-4 & P85090 & L. ocellatus & GLLDFVTGVGKDIFAQLIKQI & $\begin{array}{c}\text { E.coli } \\
\text { S.aureus }\end{array}$ & {$[14,15]$} \\
\hline 5 & $\begin{array}{l}\text { Ocellatin-4 } \\
\text { analogue }\end{array}$ & -NA- & L. ocellatus & KLLKFVTKVGKAIFKALIKAI & $\begin{array}{c}\text { X. citri } \\
\text { S. meliloti }\end{array}$ & [41] \\
\hline & \multicolumn{5}{|c|}{ https://biointerfaceresearch.com/ } & \\
\hline
\end{tabular}




\begin{tabular}{|c|c|c|c|c|c|c|}
\hline S.No & $\begin{array}{c}\text { Peptide } \\
\text { name }\end{array}$ & $\begin{array}{l}\text { UNIPROT } \\
\text { ID }\end{array}$ & Species & Sequence & Active against & Reference \\
\hline & & & & & A. tumefaciens & \\
\hline 6 & Ocellatin-5 & P85443 & L. ocellatus & GLLDFLKAAGKGLVTNL & $\begin{array}{c}\text { E.coli } \\
\text { S.aureus }\end{array}$ & [14] \\
\hline 7 & Ocellatin-6 & -NA- & L. ocellatus & AVLDFIKAAGKGLVTNIMEKVG & $\begin{array}{c}\text { E.coli } \\
\text { S.aureus }\end{array}$ & {$[16]$} \\
\hline 8 & Ocellatin-7 & -NA- & L. latrans & GVVDILKDTGKKLLSHLMEKIG & -NA- & [24] \\
\hline 9 & Ocellatin-8 & -NA- & L. latrans & GVVDILKDTGKKLLSHLMEKVG & -NA- & [24] \\
\hline 10 & Ocellatin-9 & -NA- & L. latrans & GVLDIFKDTGKKLLSHLMEKVG & -NA- & [24] \\
\hline 11 & Ocellatin-10 & -NA- & L. latrans & GLLDFLKAAGKGLVSNLIEKVG & -NA- & [24] \\
\hline 12 & Ocellatin-11 & -NA- & L. latrans & GVLDIFKDAAKQILAHAAEKIG & -NA- & [24] \\
\hline 13 & Ocellatin-F1 & P0DQJ8 & L. fallax & GVVDILKGAAKDIAGHLASKVMNKL & $\begin{array}{c}\text { E.coli } \\
\text { P.aeruginosa } \\
\text { E.cloacae } \\
\text { K.pneumoniae } \\
\end{array}$ & {$[21,22,43]$} \\
\hline 14 & $\begin{array}{l}\text { Ocellatin- } \\
\text { F1(1-22) }\end{array}$ & C0HKF1 & L. labyrinthicus & GVVDILKGAAKDIAGHLASKVM & $\begin{array}{c}\text { A.actinomycetemco } \\
\text { mitans }\end{array}$ & {$[21,22]$} \\
\hline 15 & $\begin{array}{l}\text { Ocellatin- } \\
\text { F1(1-23) }\end{array}$ & $\mathrm{COHKF} 2$ & L. labyrinthicus & GVVDILKGAAKDIAGHLASKVMN & $\begin{array}{c}\text { A.actinomycetemco } \\
\text { mitans }\end{array}$ & {$[21,22]$} \\
\hline 16 & Ocellatin-K1 & P86711 & L. knudseni & GVVDILKGAAKDLAGHLASKVMNKI & -NA- & {$[25,40]$} \\
\hline 17 & $\begin{array}{l}\text { Ocellatin- } \\
\text { K1(1-16) }\end{array}$ & -NA- & L. vastus & GVVDILKGAAKDLAGH & -NA- & [25] \\
\hline 18 & $\begin{array}{l}\text { Ocellatin- } \\
\text { K1(1-21) }\end{array}$ & -NA- & L. vastus & GVVDILKGAAKDLAGHLASKV & -NA- & [25] \\
\hline 19 & Ocellatin-1I & -NA- & L. insularum & GLLDLLKGAGKGLLTHLASQI & $\begin{array}{c}\text { E.coli } \\
\text { K.pneumoniae } \\
\text { P.aeruginosa } \\
\text { S.typhimurium }\end{array}$ & [26] \\
\hline 20 & $\begin{array}{l}\text { Ocellatin- } \\
1 \mathrm{I}(1-16)\end{array}$ & -NA- & L. insularum & GLLDLLKGAGKGLLTH & -NA- & [26] \\
\hline 21 & Ocellatin-2I & -NA- & L. insularum & GLLDFFKGAGKELLTHLASQI & $\begin{array}{c}\text { E.coli } \\
\text { K.pneumoniae } \\
\text { P.aeruginosa } \\
\text { S.typhimurium }\end{array}$ & [26] \\
\hline 22 & $\begin{array}{l}\text { Ocellatin- } \\
2 \mathrm{I}(1-16)\end{array}$ & -NA- & L. insularum & GLLDFFKGAGKELLTH & -NA- & [26] \\
\hline 23 & Ocellatin-3I & -NA- & L. insularum & GVIDILKSLGKNILTNLASKLSDNTA & -NA- & [26] \\
\hline 24 & Ocellatin-1N & -NA- & L. nesiotus & GAVVDILKGAGKNLLSLALNKLSEKV & $\begin{array}{c}\text { E.coli } \\
\text { K.pneumoniae } \\
\text { P.aeruginosa } \\
\text { S.typhimurium } \\
\end{array}$ & [26] \\
\hline 25 & Ocellatin-2N & -NA- & L. nesiotus & GAVVDILKDTGKNLLSLALNKLSEKV & -NA- & [26] \\
\hline 26 & Ocellatin-3N & -NA- & L. nesiotus & GIFDVLKNLAKGVITSLAS & $\begin{array}{c}\text { E.coli } \\
\text { S.aureus } \\
\text { K.pneumoniae } \\
\text { P.aeruginosa } \\
\text { S.typhimurium } \\
\text { E. faecium }\end{array}$ & [26] \\
\hline 27 & Ocellatin-4N & -NA- & L. nesiotus & GLFDVLKNLAKGVITSLAS & -NA- & [26] \\
\hline 28 & Ocellatin-S1 & P85279 & L. syphax & GVLDILKGAAKDLAGHVATKVINKI & $\begin{array}{c}\text { E.coli } \\
\text { S.aureus }\end{array}$ & [18] \\
\hline 29 & Ocellatin-L1 & P0DQL0 & L. laticeps & GVVDILKGAAKDLAGHLATKVMNKL & $\begin{array}{c}\text { E.coli } \\
\text { S.aureus }\end{array}$ & {$[20]$} \\
\hline 30 & Ocellatin-L2 & P0DQL1 & L. laticeps & GVVDILKGAAKDLAGHLATKVMDKL & -NA- & [20] \\
\hline 31 & $\begin{array}{l}\text { Ocellatin- } \\
\text { PT1 }\end{array}$ & C0HJZ6 & L. pustulatus & $\begin{array}{c}\text { MAFLKKSLFLVLFLGLVSLSICDEEKR } \\
\text { QDEDDDDDDDEEKRGVFDIIKDAGKQ } \\
\text { LVAHAMGKIAEKV }\end{array}$ & $\begin{array}{c}\text { E.coli } \\
\text { S.aureus } \\
\text { K.pneumoniae } \\
\text { S.choleraesuis }\end{array}$ & [17] \\
\hline 32 & $\begin{array}{l}\text { Ocellatin- } \\
\text { PT2 }\end{array}$ & $\mathrm{COHJZ7}$ & L. pustulatus & $\begin{array}{l}\text { MAFLKKSLFLVLFLGLVSLSICDEEKR } \\
\text { QDEDDDDDDDEEKRGVFDIIKDAGKQ } \\
\text { LVAHATGKIAEKV }\end{array}$ & -NA- & [17] \\
\hline 33 & $\begin{array}{l}\text { Ocellatin- } \\
\text { PT3 }\end{array}$ & $\mathrm{COHJZ8}$ & L. pustulatus & $\begin{array}{c}\text { MAFLKKSLFLVLFLGLVSLSICDEEKR } \\
\text { QDEDDDDDDDEEKRGVIDIIKGAGKD } \\
\text { LIAHAIG } \\
\text { KLAEKV }\end{array}$ & $\begin{array}{c}\text { E.coli } \\
\text { S.aureus } \\
\text { K.pneumoniae } \\
\text { S.choleraesuis } \\
\text { P.aeruginosa }\end{array}$ & {$[17]$} \\
\hline
\end{tabular}




\begin{tabular}{|c|c|c|c|c|c|c|}
\hline S.No & $\begin{array}{l}\text { Peptide } \\
\text { name }\end{array}$ & $\begin{array}{l}\text { UNIPROT } \\
\text { ID }\end{array}$ & Species & Sequence & Active against & Reference \\
\hline 34 & $\begin{array}{l}\text { Ocellatin- } \\
\text { PT4 }\end{array}$ & COHJZ9 & L. pustulatus & $\begin{array}{l}\text { MAFLKKSLFLVLFLGLVSLSICDEEKR } \\
\text { QDEDDDDDDDEEKRGVFDIIKGAGKQ } \\
\text { LIAHAMGKIAEKV }\end{array}$ & $\begin{array}{l}\text { E.coli } \\
\text { S.aureus } \\
\text { K.pneumoniae } \\
\text { S.choleraesuis }\end{array}$ & [17] \\
\hline 35 & $\begin{array}{l}\text { Ocellatin- } \\
\text { PT5 }\end{array}$ & $\mathrm{COHK00}$ & L. pustulatus & $\begin{array}{l}\text { MAFLKKSLFLVLFLGLVSLSICDEEKR } \\
\text { QDEDDDDDDDEEKRGVFDIIKDAGRQ } \\
\text { LVAHAMGKIAEKV }\end{array}$ & $\begin{array}{c}\text { E.coli } \\
\text { S.aureus, } \\
\text { K.pneumoniae } \\
\text { S.choleraesuis }\end{array}$ & {$[17]$} \\
\hline 36 & $\begin{array}{l}\text { Ocellatin- } \\
\text { PT6 }\end{array}$ & $\mathrm{C} 0 \mathrm{HK} 01$ & L. pustulatus & $\begin{array}{l}\text { MAFLKKSLFLVLFLGLVSLSICDEEKR } \\
\text { QDEDDDDDDDEEKRGVFDIIKGAGKQ } \\
\text { LIAHAMEKIAEKVGLNKDGN }\end{array}$ & $\begin{array}{l}\text { E.coli } \\
\text { S.aureus, } \\
\text { K.pneumoniae } \\
\text { S.choleraesuis }\end{array}$ & {$[17]$} \\
\hline 37 & $\begin{array}{l}\text { Ocellatin- } \\
\text { PT7 }\end{array}$ & $\mathrm{COHK02}$ & L. pustulatus & $\begin{array}{l}\text { MAFLKKSLFLVLFLGLVSLSICDEEKR } \\
\text { QDEDDDDDDDEEKRGVFDIIKGAGKQ } \\
\text { LIAHAMGKIAEKVGLNKDGN }\end{array}$ & $\begin{array}{l}\quad \text { E.coli } \\
\quad \text { S.aureus } \\
\text { K.pneumoniae } \\
\text { S.choleraesuis }\end{array}$ & {$[17]$} \\
\hline 38 & $\begin{array}{l}\text { Ocellatin- } \\
\text { PT8 }\end{array}$ & $\mathrm{COHK03}$ & L. pustulatus & $\begin{array}{l}\text { MAFLKKSLFLVLFLGLVSLSICDEEKR } \\
\text { QDEDDDDDDDEEKRGVFDIIKGAGKQ } \\
\text { LIARAMGKIAEKVGLNKDGN }\end{array}$ & $\begin{array}{l}\text { E.coli } \\
\text { S.aureus } \\
\text { K.pneumoniae } \\
\text { S.choleraesuis }\end{array}$ & [17] \\
\hline 39 & Ocellatin-P1 & P0DQJ7 & L. pentadactylus & GLLDTLKGAAKNVVGSLASKVMEKL & $\begin{array}{c}\text { E.coli } \\
\text { E. cloacae } \\
\text { K.pneumoniae } \\
\text { P.aeruginosa } \\
\text { S.aureus } \\
\text { S. epidermidis } \\
\text { E. faecalis }\end{array}$ & [43] \\
\hline 40 & Ocellatin-V1 & -NA- & L. validus & GVVDILKGAGKDLLAHALSKLSEKV & $\begin{array}{c}\text { E.coli } \\
\text { S.aureus } \\
\end{array}$ & [19] \\
\hline 41 & Ocellatin-V2 & -NA- & L. validus & GVLDILKGAGKDLLAHALSKISEKV & $\begin{array}{c}\text { E.coli } \\
\text { S.aureus }\end{array}$ & [19] \\
\hline 42 & Ocellatin-V3 & -NA- & L. validus & GVLDILTGAGKDLLAHALSKLSEKV & $\begin{array}{c}\text { E.coli } \\
\text { S.aureus }\end{array}$ & [19] \\
\hline
\end{tabular}

-NA- Not Applicable

\subsection{Gram negative bacteria.}

Gram-negative bacteria like E.coli and K. pneumoniae are common in our gut as commensals and do not affect humans. However, they begin as primary food contamination and ensure multi-organ diseases affecting the lungs, brain, heart, and urinary tract. In which nearly all Ocellatin peptides inhibit E.coli (Table 1). Conversely, Ocellatin-F1 and OcellatinPT1-PT8 excluding PT2 inhibited carbapenem-resistant $K$. pneumoniae bacteria producing lung illness [42, 43]. Salmonella typhimurium (S. typhimurium), a food-borne pathogen, is resistant to antibiotics such as chloramphenicol, tetracycline, cephalosporins, sulfonamides, and streptomycin [44]. Owing to its multidrug-resistant nature, typhoid and associated gastroenteritis in humans turn challenging to treat [45]. However, Ocellatin 1N, 1I, 2I, 3N exhibited resistance against $S$. typhimurium with minimum inhibition concentration (MIC) at the range of $31.25-62.5 \mu \mathrm{M}$.

Another opportunistic Gram-negative bacterium resistant to many drugs is Pseudomonas aeruginosa ( $P$. aeruginosa) [46]. The evolved communication mechanisms in $P$. aeruginosa, besides quorum sensing, endorsed it to develop biofilms [47,48]. However, AMPs act as a new class of anti-biofilm compounds since they can either restrict biofilm formation or remove established biofilms [49,50]. P. aeruginosa can be inhibited with Ocellatin PT3 (MIC=16 $\mu \mathrm{g} / \mathrm{ml}$ ), which is the most effective of the extensively investigated Ocellatin peptides (PT2-PT6) [51]. 


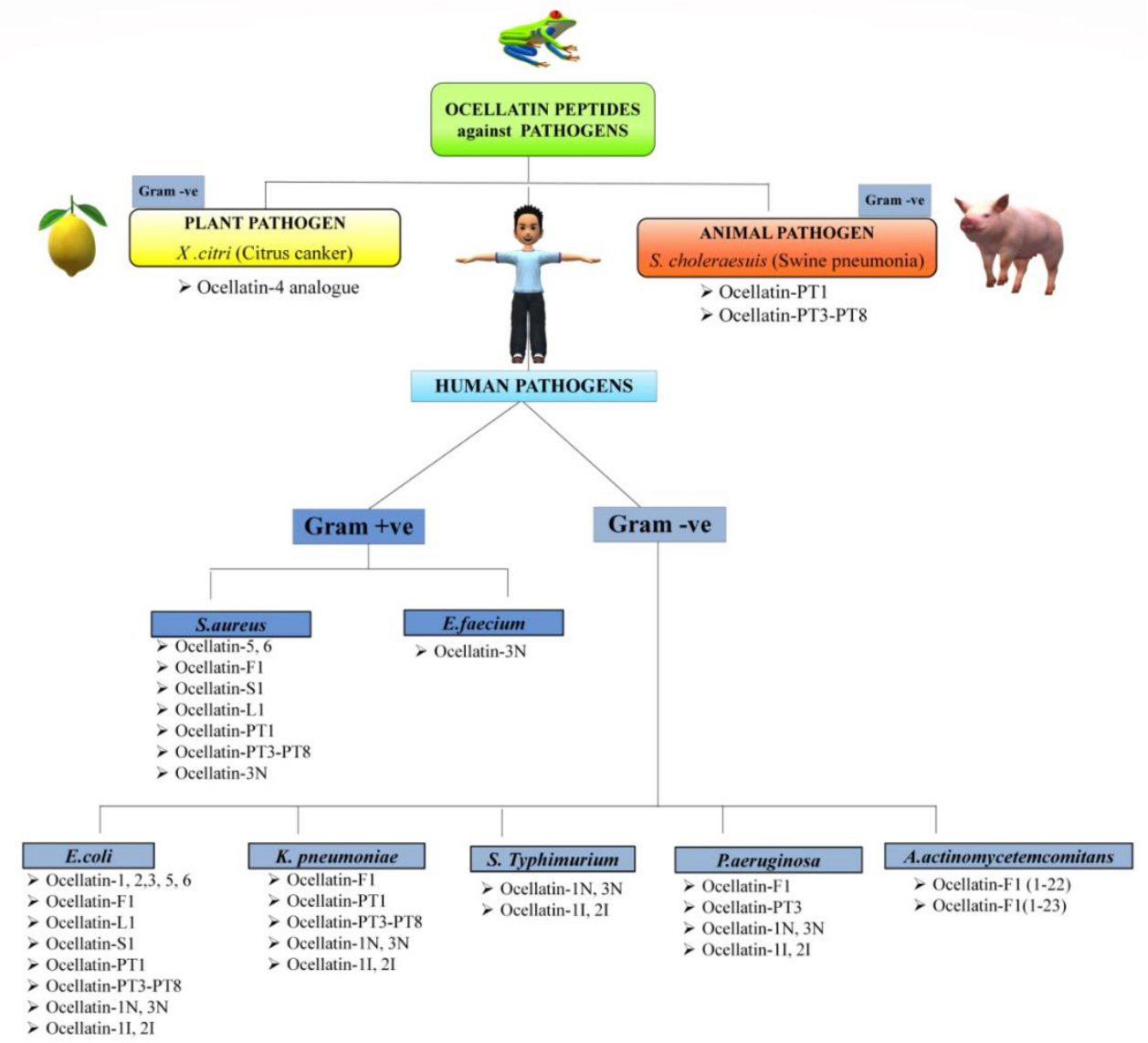

Figure 2. A schematic representation depicting Ocellatin peptides and three major forms of diseases they combat.

S. choleraesuis is yet another Gram-negative bacterium that causes swine pneumonia in pigs and affects the pork supply chain [52]. Moreover, the contaminated pork poses a health risk to humans. Because it causes illness in pigs and humans, the economic cost is doubled by S. choleraesuis [53]. Ocellatin-PT1 and PT3-PT8 may mitigate Salmonella infection, whereas Ocellatin-PT2 binds to lipopolysaccharide (LPS) at first, but the peptide cannot suppress Gramnegative bacterial growth [51,54]. Xanthomonas citri (X. citri), a common phytopathogen, is a rod-shaped Gram-negative bacterium [55,56]. Interestingly, analogs of Ocellatin-4 have been proven to be potent against $X$. citri, preventing citrus fruit damage and possibly increasing the economic value. Aggregatibacter actinomycetemcomitans (A. actinomycetemcomitans) is another Gram-negative facultative bacterium that can cause aggressive dental gum inflammation (periodontitis) [57]. Periodontitis may be caused by non-infectious risk factors like smoking, stress, and aging, and hormonal changes in addition to bacterial infection that result in dental biofilms [58,59]. However, Ocellatin-F1 (1-22) and Ocellatin-F1 (1-23), commonly known as Ocellatin-LB1 and Ocellatin-LB2, are employed to treat periodontitis.

\subsection{Gram-positive bacteria.}

The gram-positive bacterium, Staphylococcus aureus (S. aureus), causing multi-organ illness, is still a serious public health problem, as it causes nosocomial (hospital-acquired) infections [60]. Ocellatin-3N, Ocellatin-5, Ocellatin-6, Ocellatin-F1, Ocellatin-S1, OcellatinL1, and Ocellatin-PT1-PT8, all curbed methicillin-resistant S. aureus. Enterococcus faecium, a Gram-positive gut commensal, causes a significant proportion of hospital-acquired infections with high mortality [61]. Ocellatin-3N, on the other hand, acts potently against that bacteria. 


\section{Therapeutic Modifications to Enhance Antibacterial Potential}

One of the essential post-translational modifications in any alpha-helical AMP is Cterminal amidation in which the hydroxyl $(\mathrm{OH})$ group is substituted with the amide $(\mathrm{NH} 2)$ group at $\mathrm{C}$-terminus $[62,63]$. C-terminal amidation regulates peptide stability and functionality in various ways viz., i) stabilizing helicity ii) increasing cationic iii) altering dipole moment iv) degenerating carboxypeptidases and thereby, (v) increasing bactericidal activity [64]. Cterminal amidation aspects have been reported to be highly effective in Ocellatin peptides such as Ocellatin-PT1 to Ocellatin-PT5, Ocellatin-5, and Ocellatin-6 (Figure 3).

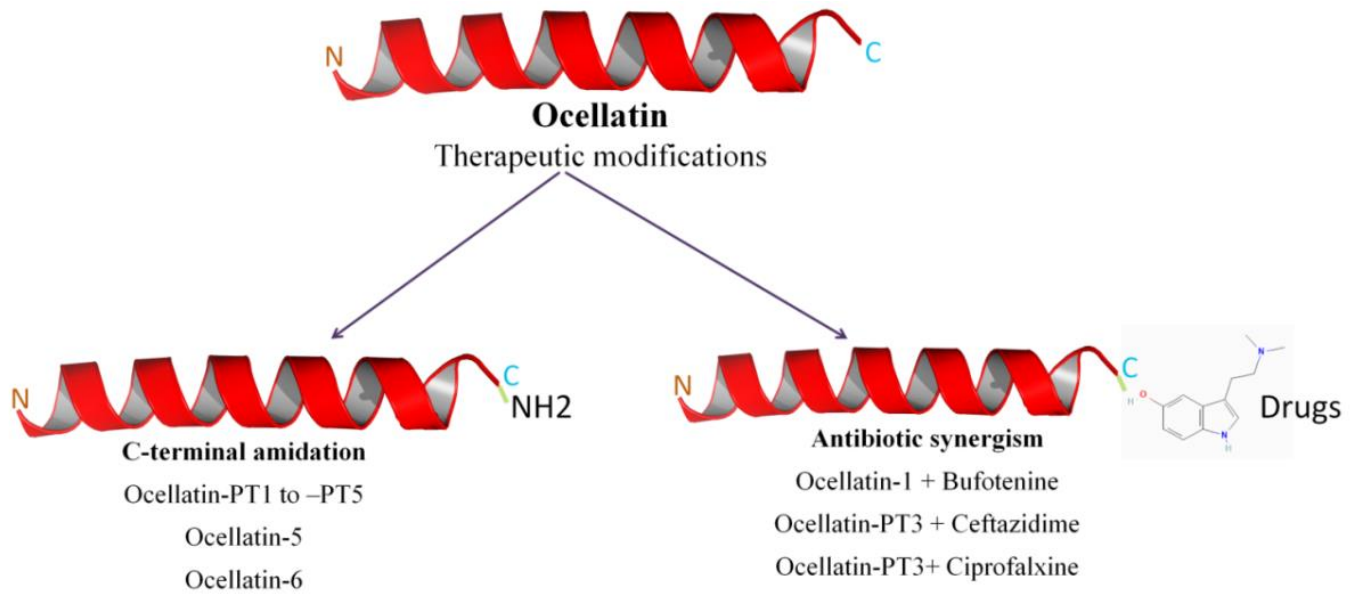

Figure 3. The illustration of therapeutic modifications in Ocellatin peptides notably, C-terminal amidation and antibacterial synergism.

Synergizing the effects of antibiotics with AMPs is beneficial for combating multidrugresistant bacteria [65]. Ceftazidime (cephalosporin antibiotic) and Ciproflaxine (quinolone antibiotic) have been synergized with Ocellatin-PT3. With the help of Ocellatin-PT3, these alkaloid drugs have substantially improved their permeability of cell membranes of multi-drug resistant (MDR) isolates. Moreover, for evaluating antiviral activity against the Rabies virus, Ocellatin-1 was conjugated with the serotonin-derived alkaloid Bufotenine [66].

\section{Limitations and Future Perspectives}

There are a few drawbacks of using AMPs on a wide scale. Mostly alpha-helical AMPs are degraded by extracellular proteinases due to their co-evolution with AMP-producing hosts. Henceforth, the pathogens also develop various resistance mechanisms such as the loss of activity at human physiological salt concentrations and increased hemolytic effect cleaving human erythrocytes. Another important limitation in using AMP like Ocellatin is the lack of appropriate strategies to deliver drugs sustainably [67].

Pertinently, this could be avoided in the future by incorporating D-form non-natural amino acids or by employing non-peptide backbones via peptidomimetics, which could lead to the development of alpha-helical AMPs as potential antimicrobial drugs. Particularly, D-amino substitutions will reduce the hemolytic activity by increasing the antibacterial activity simultaneously $[68,69]$. Further, understanding the supramolecular chemistry of peptide selfassembly can better understand how biofilms and secondary targets function, leading to new directions in the formulation of self-assembled Ocellatin peptide nanostructures in therapeutics. Furthermore, the emergence of new big data design methods like machine 
learning will facilitate researchers to explore new targets for Ocellatin AMPs other than the membrane.

\section{Conclusions}

To summarise, the entire globe seeks novel drugs to combat alarming antimicrobial resistance and antibiotic failure. However, the alpha-helical AMPs isolated from multi-habitat organisms such as frogs would be a preferable alternative for the pharmaceutical industry to employ AMPs as medications in the future. Considering the versatility of Ocellatin AMPs, they can be used in animal husbandry and agricultural sectors in addition to clinical applications. We anticipate that this review article will help pharmaceutical and agricultural researchers to understand the potential of Ocellatin.

\section{Funding}

The authors thank VIT Deemed to be University for providing 'VIT SEED GRANT (VIT/SG/2020-21/43)' for carrying out this review.

\section{Acknowledgments}

We thank VIT Deemed to be University for their infrastructure support in compiling this review article.

\section{Conflicts of Interest}

The authors declare no conflict of interest.

\section{References}

1. Gaynes, R. The Discovery of Penicillin-New Insights After More Than 75 Years of Clinical Use. Emerg. Infect. Dis. 2017, 23, 849-853, https://doi.org/10.3201/eid2305.161556.

2. Kasimanickam, V.; Kasimanickam, M.; Kasimanickam, R. Antibiotics Use in Food Animal Production: Escalation of Antimicrobial Resistance: Where Are We Now in Combating AMR? Medical Sciences 2021, 9, https://doi.org/10.3390/medsci9010014.

3. Kumar, M.; Sarma, D.K.; Shubham, S.; Kumawat, M.; Verma, V.; Nina, P.B.; Jp, D.; Kumar, S.; Singh, B.; Tiwari, R.R. Futuristic Non-Antibiotic Therapies to Combat Antibiotic Resistance: A Review. Front. Microbiol. 2021, 12, https://doi.org/10.3389/fmicb.2021.609459.

4. Zaman, S.B.; Hussain, M.A.; Nye, R.; Mehta, V.; Mamun, K.T.; Hossain, N. A Review on Antibiotic Resistance: Alarm Bells Are Ringing. Cureus 2017, https://doi.org/10.7759/cureus.1403.

5. Gan, B.H.; Gaynord, J.; Rowe, S.M.; Deingruber, T.; Spring, D.R. The Multifaceted Nature of Antimicrobial Peptides: Current Synthetic Chemistry Approaches and Future Directions. Chem. Soc. Rev. 2021, 50, 78207880, https://doi.org/10.1039/D0CS00729C.

6. Hancock, R.E.W.; Diamond, G. The Role of Cationic Antimicrobial Peptides in Innate Host Defences. Trends in Microbiology 2000, 8, 402-410, https://doi.org/10.1016/S0966-842X(00)01823-0.

7. Mahlapuu, M.; Håkansson, J.; Ringstad, L.; Björn, C. Antimicrobial Peptides: An Emerging Category of Therapeutic Agents. Front. Cell. Infect. Microbiol. 2016, 6, https://doi.org/10.3389/fcimb.2016.00194.

8. Rončević, T.; Puizina, J.; Tossi, A. Antimicrobial Peptides as Anti-Infective Agents in Pre-Post-Antibiotic Era? IJMS 2019, 20, https://doi.org/10.3390/ijms20225713.

9. Cardoso, P.; Glossop, H.; Meikle, T.G.; Aburto-Medina, A.; Conn, C.E.; Sarojini, V.; Valery, C. Molecular Engineering of Antimicrobial Peptides: Microbial Targets, Peptide Motifs and Translation Opportunities. Biophys Rev 2021, 13, 35-69, https://doi.org/10.1007/s12551-021-00784-y.

10. Coates, M.; Blanchard, S.; MacLeod, A.S. Innate Antimicrobial Immunity in the Skin: A Protective Barrier against Bacteria, Viruses, and Fungi. PLoS Pathog 2018, 14, https://doi.org/10.1371/journal.ppat.1007353.

11. Varga, J.F.A.; Bui-Marinos, M.P.; Katzenback, B.A. Frog Skin Innate Immune Defences: Sensing and Surviving Pathogens. Front. Immunol. 2019, 9, https://doi.org/10.3389/fimmu.2018.03128. 
12. Wang, Y.; Liu, M.; Gao, J. Enhanced Receptor Binding of SARS-CoV-2 through Networks of HydrogenBonding and Hydrophobic Interactions. Proc Natl Acad Sci USA 2020, 117, 13967-13974, https://doi.org/10.1073/pnas.2008209117.

13. Cunha Neto, R. dos S.; Vigerelli, H.; Jared, C.; Antoniazzi, M.M.; Chaves, L.B.; da Silva, A. de C.R.; Melo, R.L. de; Sciani, J.M.; Pimenta, D.C. Synergic Effects between Ocellatin-F1 and Bufotenine on the Inhibition of BHK-21 Cellular Infection by the Rabies Virus. J Venom Anim Toxins Incl Trop Dis 2015, 21.

14. Nascimento, A.C.C.; Zanotta, L.C.; Kyaw, C.M.; Schwartz, E.N.F.; Schwartz, C.A.; Sebben, A.; Sousa, M.V.; Fontes, W.; Castro, M.S. Ocellatins: New Antimicrobial Peptides from the Skin Secretion of the South American Frog Leptodactylus Ocellatus (Anura: Leptodactylidae). Protein J 2004, 23, 501-508, https://doi.org/10.1007/s10930-004-7877-z.

15. Nascimento, A.; Chapeaurouge, A.; Perales, J.; Sebben, A.; Sousa, M.V.; Fontes, W.; Castro, M.S. Purification, Characterization and Homology Analysis of Ocellatin 4, a Cytolytic Peptide from the Skin Secretion of the Frog Leptodactylus Ocellatus. Toxicon 2007, 50, 1095-1104, https://doi.org/10.1016/j.toxicon.2007.07.014.

16. Leite, J.M.A.; Silva, L.P.; Silva-Leite, R.R.; Ferrari, A.S.; Noronha, S.E.; Silva, H.R.; Bloch, C.; Leite, J.R.d.S.d.A. Leptodactylus Ocellatus (Amphibia): Mechanism of Defense in the Skin and Molecular Phylogenetic Relationships. J Exp Zool A Ecol Genet Physiol 2010, 313, 1-8, https://doi.org/10.1002/jez.551.

17. Marani, M.M.; Dourado, F.S.; Quelemes, P.V.; de Araujo, A.R.; Perfeito, M.L.G.; Barbosa, E.A.; Véras, L.M.C.; Coelho, A.L.R.; Andrade, E.B.; Eaton, P.; Longo, J.P.F.; Azevedo, R.B.; Delerue-Matos, C.; Leite, J.R.S.A. Characterization and Biological Activities of Ocellatin Peptides from the Skin Secretion of the Frog Leptodactylus Pustulatus. J. Nat. Prod. 2015, 78, 1495-1504, https://doi.org/10.1021/np500907t.

18. Dourado, F.S.; Leite, J.R.S.A.; Silva, L.P.; Melo, J.A.T.; Bloch, C.; Schwartz, E.F. Antimicrobial Peptide from the Skin Secretion of the Frog Leptodactylus Syphax. Toxicon 2007, 50, 572-580, https://doi.org/10.1016/j.toxicon.2007.04.027.

19. King, J.D.; Leprince, J.; Vaudry, H.; Coquet, L.; Jouenne, T.; Conlon, J.M. Purification and Characterization of Antimicrobial Peptides from the Caribbean Frog, Leptodactylus Validus (Anura: Leptodactylidae). Peptides 2008, 29, 1287-1292, https://doi.org/10.1016/j.peptides.2008.04.005.

20. Conlon, J.M.; Abdel-Wahab, Y.H.A.; Flatt, P.R.; Leprince, J.; Vaudry, H.; Jouenne, T.; Condamine, E. A Glycine-Leucine-Rich Peptide Structurally Related to the Plasticins from Skin Secretions of the Frog Leptodactylus Laticeps (Leptodactylidae). Peptides $\quad$ 2009, $\quad 30, \quad 888-892$, https://doi.org/10.1016/j.peptides.2009.01.008.

21. Gusmão, K.A.G.; dos Santos, D.M.; Santos, V.M.; Cortés, M.E.; Reis, P.V.M.; Santos, V.L.; Piló-Veloso, D.; Verly, R.M.; de Lima, M.E.; Resende, J.M. Ocellatin Peptides from the Skin Secretion of the South American Frog Leptodactylus Labyrinthicus (Leptodactylidae): Characterization, Antimicrobial Activities and Membrane Interactions. J Venom Anim Toxins Incl Trop Dis 2017, 23, https://doi.org/10.1186/s40409017-0094-y.

22. Gomes, K.A.G.G.; dos Santos, D.M.; Santos, V.M.; Piló-Veloso, D.; Mundim, H.M.; Rodrigues, L.V.; Lião, L.M.; Verly, R.M.; de Lima, M.E.; Resende, J.M. NMR Structures in Different Membrane Environments of Three Ocellatin Peptides Isolated from Leptodactylus Labyrinthicus. Peptides 2018, 103, 72-83, https://doi.org/10.1016/j.peptides.2018.03.016.

23. Conlon, J.M. A Proposed Nomenclature for Antimicrobial Peptides from Frogs of the Genus Leptodactylus. Peptides 2008, 29, 1631-1632, https://doi.org/10.1016/j.peptides.2008.04.016.

24. Marani, M.M.; Aguilar, S.; Cuzziol Boccioni, A.P.; Cancelarich, N.L.; Basso, N.G.; Albericio, F. Identification of New Ocellatin Antimicrobial Peptides by CDNA Precursor Cloning in the Frame of This Family of Intriguing Peptides. Antibiotics 2020, 9, https://doi.org/10.3390/antibiotics9110751.

25. Sousa, N.A.; Oliveira, G.A.L.; de Oliveira, A.P.; Lopes, A.L.F.; Iles, B.; Nogueira, K.M.; Araújo, T.S.L.; Souza, L.K.M.; Araújo, A.R.; Ramos-Jesus, J.; Plácido, A.; Amaral, C.; Campelo, Y.D.M.; Barbosa, E.A.; Portugal, C.C.; Socodato, R.; Lobo, A.; Relvas, J.; Bemquerer, M.; Eaton, P.; Leite, J.R.S.A.; Medeiros, J.V.R. Novel Ocellatin Peptides Mitigate LPS-Induced ROS Formation and NF-KB Activation in Microglia and Hippocampal Neurons. Sci Rep 2020, 10, 2696, https://doi.org/10.1038/s41598-020-59665-1.

26. Barran, G.; Kolodziejek, J.; Coquet, L.; Leprince, J.; Jouenne, T.; Nowotny, N.; Conlon, J.M.; Mechkarska, M. Peptidomic Analysis of Skin Secretions of the Caribbean Frogs Leptodactylus Insularum and Leptodactylus Nesiotus (Leptodactylidae) Identifies an Ocellatin with Broad Spectrum Antimicrobial Activity. Antibiotics 2020, 9, https://doi.org/10.3390/antibiotics9100718.

27. Yan, Y.; Li, Y.; Zhang, Z.; Wang, X.; Niu, Y.; Zhang, S.; Xu, W.; Ren, C. Advances of Peptides for Antibacterial Applications. Colloids Surf B Biointerfaces 2021, 202, https://doi.org/10.1016/j.colsurfb.2021.111682.

28. Moretta, A.; Scieuzo, C.; Petrone, A.M.; Salvia, R.; Manniello, M.D.; Franco, A.; Lucchetti, D.; Vassallo, A.; Vogel, H.; Sgambato, A.; Falabella, P. Antimicrobial Peptides: A New Hope in Biomedical and Pharmaceutical Fields. Front. Cell. Infect. Microbiol. 2021, 11, 668632, https://doi.org/10.3389/fcimb.2021.668632. 
29. Omardien, S.; Brul, S.; Zaat, S.A.J. Antimicrobial Activity of Cationic Antimicrobial Peptides against GramPositives: Current Progress Made in Understanding the Mode of Action and the Response of Bacteria. Front. Cell Dev. Biol. 2016, 4, https://doi.org/10.3389/fcell.2016.00111.

30. Bhattacharjya, S.; Straus, S.K. Design, Engineering and Discovery of Novel $\alpha$-Helical and $\beta$-Boomerang Antimicrobial Peptides against Drug Resistant Bacteria. IJMS 2020, 21, 5773, https://doi.org/10.3390/ijms21165773.

31. Rai, D.K.; Qian, S. Interaction of the Antimicrobial Peptide Aurein 1.2 and Charged Lipid Bilayer. Sci Rep 2017, 7, https://doi.org/10.1038/s41598-017-03795-6.

32. Sengupta, D.; Leontiadou, H.; Mark, A.E.; Marrink, S.-J. Toroidal Pores Formed by Antimicrobial Peptides Show Significant Disorder. Biochimica et Biophysica Acta (BBA) - Biomembranes 2008, 1778, 2308-2317, https://doi.org/10.1016/j.bbamem.2008.06.007.

33. Tuerkova, A.; Kabelka, I.; Králová, T.; Sukeník, L.; Pokorná, Š.; Hof, M.; Vácha, R. Effect of Helical Kink in Antimicrobial Peptides on Membrane Pore Formation. eLife 2020, 9, https://doi.org/10.7554/eLife.47946.

34. Shi, J.; Chen, C.; Wang, D.; Tong, Z.; Wang, Z.; Liu, Y. Amphipathic Peptide Antibiotics with Potent Activity against Multidrug-Resistant Pathogens. Pharmaceutics https://doi.org/10.3390/pharmaceutics13040438.

35. Elliott, A.G.; Huang, J.X.; Neve, S.; Zuegg, J.; Edwards, I.A.; Cain, A.K.; Boinett, C.J.; Barquist, L.; Lundberg, C.V.; Steen, J.; Butler, M.S.; Mobli, M.; Porter, K.M.; Blaskovich, M.A.T.; Lociuro, S.; Strandh, M.; Cooper, M.A. An amphipathic peptide with antibiotic activity against multidrug-resistant Gram-negative bacteria. Nature Communications 2020, 11, https://doi.org/10.1038/s41467-020-16950-x.

36. He, S.; Yang, Z.; Yu, W.; Li, J.; Li, Z.; Wang, J.; Shan, A. Systematically Studying the Optimal Amino Acid Distribution Patterns of the Amphiphilic Structure by Using the Ultrashort Amphiphiles. Front. Microbiol. 2020, 11, https://doi.org/10.3389/fmicb.2020.569118.

37. Petkov, P.; Lilkova, E.; Ilieva, N.; Litov, L. Self-Association of Antimicrobial Peptides: A Molecular Dynamics Simulation Study on Bombinin. IJMS 2019, 20, https://doi.org/10.3390/ijms20215450.

38. Chandra Sekar, P.; Chandrasekhar, G.; Rajasekaran, R. Hydrophobic Residues Confer the Helicity and Membrane Permeability of Ocellatin-1 Antimicrobial Peptide Scaffold Towards Therapeutics. Int J Pept Res Ther 2021, 27, 2459-2470, https://doi.org/10.1007/s10989-021-10265-1.

39. Jiang, Z.; Vasil, A.I.; Hale, J.D.; Hancock, R.E.W.; Vasil, M.L.; Hodges, R.S. Effects of Net Charge and the Number of Positively Charged Residues on the Biological Activity of Amphipathic $\alpha$-Helical Cationic Antimicrobial Peptides. Biopolymers 2008, 90, 369-383, https://doi.org/10.1002/bip.20911.

40. Sekar, P.C.; Paul, D.M.; Srinivasan, E.; Rajasekaran, R. Unravelling the Molecular Effect of Ocellatin-1, F1, K1 and S1, the Frog-Skin Antimicrobial Peptides to Enhance Its Therapeutics - Quantum and Molecular Mechanical Approaches. J Mol Model 2021, 27, https://doi.org/10.1007/s00894-020-04652-6.

41. Inui Kishi, R.N.; Stach-Machado, D.; Singulani, J. de L.; dos Santos, C.T.; Fusco-Almeida, A.M.; Cilli, E.M.; Freitas-Astúa, J.; Picchi, S.C.; Machado, M.A. Evaluation of Cytotoxicity Features of Antimicrobial Peptides with Potential to Control Bacterial Diseases of Citrus. PLoS ONE 2018, 13, https://doi.org/10.1371/journal.pone.0203451.

42. Rollins-Smith, L.A.; King, J.D.; Nielsen, P.F.; Sonnevend, A.; Conlon, J.M. An Antimicrobial Peptide from the Skin Secretions of the Mountain Chicken Frog Leptodactylus Fallax (Anura:Leptodactylidae). Regulatory Peptides 2005, 124, 173-178, https://doi.org/10.1016/j.regpep.2004.07.013.

43. King, J.D.; Al-Ghaferi, N.; Abraham, B.; Sonnevend, A.; Leprince, J.; Nielsen, P.F.; Conlon, J.M. Pentadactylin: An Antimicrobial Peptide from the Skin Secretions of the South American Bullfrog Leptodactylus Pentadactylus. Comp Biochem Physiol C Toxicol Pharmacol 2005, 141, 393-397, https://doi.org/10.1016/j.cbpc.2005.09.002.

44. Xiang, Y.; Li, F.; Dong, N.; Tian, S.; Zhang, H.; Du, X.; Zhou, X.; Xu, X.; Yang, H.; Xie, J.; Yang, C.; Liu, H.; Qiu, S.; Song, H.; Sun, Y. Investigation of a Salmonellosis Outbreak Caused by Multidrug Resistant Salmonella Typhimurium in China. Frontiers in Microbiology 2020, 11, https://doi.org/10.3389/fmicb.2020.00801.

45. Dyson, Z.A.; Klemm, E.J.; Palmer, S.; Dougan, G. Antibiotic Resistance and Typhoid. Clinical Infectious Diseases 2019, 68, S165-S170, https://doi.org/10.1093/cid/ciy1111.

46. Kousovista, R.; Athanasiou, C.; Liaskonis, K.; Ivopoulou, O.; Karalis, V. Association of Antibiotic Use with the Resistance Epidemiology of Pseudomonas Aeruginosa in a Hospital Setting: A Four-Year Retrospective Time Series Analysis. Sci. Pharm. 2021, 89, https://doi.org/10.3390/scipharm89010013.

47. Thi, M.T.T.; Wibowo, D.; Rehm, B.H.A. Pseudomonas Aeruginosa Biofilms. Int. J. Mol. Sci. 2020, 21, 8671, https://doi.org/10.3390/ijms21228671.

48. Moradali, M.F.; Ghods, S.; Rehm, B.H.A. Pseudomonas Aeruginosa Lifestyle: A Paradigm for Adaptation, Survival, and Persistence. Front. Cell. Infect. Microbiol. 2017, 7, https://doi.org/10.3389/fcimb.2017.00039.

49. Muhammad, M.H.; Idris, A.L.; Fan, X.; Guo, Y.; Yu, Y.; Jin, X.; Qiu, J.; Guan, X.; Huang, T. Beyond Risk: Bacterial Biofilms and Their Regulating Approaches. Front. Microbiol. 2020, 11, https://doi.org/10.3389/fmicb.2020.00928.

50. Di Somma, A.; Moretta, A.; Canè, C.; Cirillo, A.; Duilio, A. Antimicrobial and Antibiofilm Peptides. Biomolecules 2020, 10, https://doi.org/10.3390/biom10040652. 
51. Bessa, L.J.; Eaton, P.; Dematei, A.; Plácido, A.; Vale, N.; Gomes, P.; Delerue-Matos, C.; SA Leite, J.R.; Gameiro, P. Synergistic and Antibiofilm Properties of Ocellatin Peptides against Multidrug-Resistant Pseudomonas Aeruginosa. Future Microbiology 2018, 13, 151-163, https://doi.org/10.2217/fmb-2017-0175.

52. Leekitcharoenphon, P.; Sørensen, G.; Löfström, C.; Battisti, A.; Szabo, I.; Wasyl, D.; Slowey, R.; Zhao, S.; Brisabois, A.; Kornschober, C.; Kärssin, A.; Szilárd, J.; Černý, T.; Svendsen, C.A.; Pedersen, K.; Aarestrup, F.M.; Hendriksen, R.S. Cross-Border Transmission of Salmonella Choleraesuis Var. Kunzendorf in European Pigs and Wild Boar: Infection, Genetics, and Evolution. Front. Microbiol. 2019, 10, https://doi.org/10.3389/fmicb.2019.00179.

53. Eng, S.-K.; Pusparajah, P.; Ab Mutalib, N.-S.; Ser, H.-L.; Chan, K.-G.; Lee, L.-H. Salmonella: A Review on Pathogenesis, Epidemiology and Antibiotic Resistance. Frontiers in Life Science 2015, 8, 284-293, https://doi.org/10.1080/21553769.2015.1051243.

54. Oliveira, M.; Gomes-Alves, A.G.; Sousa, C.; Mirta Marani, M.; Plácido, A.; Vale, N.; Delerue-Matos, C.; Gameiro, P.; Kückelhaus, S.A.S.; Tomas, A.M.; S. A. Leite, J.R.; Eaton, P. Ocellatin-PT antimicrobial peptides: High-resolution microscopy studies in antileishmania models and interactions with mimetic membrane systems. Biopolymers 2016, 105, 873-886, https://doi.org/10.1002/bip.22925.

55. Martins, P.M.M.; Wood, T.K.; de Souza, A.A. Persister Cells Form in the Plant Pathogen Xanthomonas Citri Subsp. Citri under Different Stress Conditions. Microorganisms 2021, 9, https://doi.org/10.3390/microorganisms9020384.

56. Pruvost, O.; Richard, D.; Boyer, K.; Javegny, S.; Boyer, C.; Chiroleu, F.; Grygiel, P.; Parvedy, E.; Robène, I.; Maillot-Lebon, V.; Hamza, A.; Lobin, K.K.; Naiken, M.; Vernière, C. Diversity and Geographical Structure of Xanthomonas Citri Pv. Citri on Citrus in the South West Indian Ocean Region. Microorganisms 2021, 9, 945, https://doi.org/10.3390/microorganisms9050945.

57. Ozuna, H.; Uriarte, S.M.; Demuth, D.R. The Hunger Games: Aggregatibacter Actinomycetemcomitans Exploits Human Neutrophils As an Epinephrine Source for Survival. Front. Immunol. 2021, 12, https://doi.org/10.3389/fimmu.2021.707096.

58. Oscarsson, J.; DiRienzo, J.; Johansson, A. Editorial Comments to the Special Issue: “Aggregatibacter Actinomycetemcomitans - Gram-Negative Bacterial Pathogen.” Pathogens 2020, 9, https://doi.org/10.3390/pathogens9060441.

59. Kriswandini, I.L.; I, D.; Tantiana; P, N.; T, B.; Ia, P. The Forming of Bacteria Biofilm from Streptococcus Mutans and Aggregatibacter Actinomycetemcomitans as a Marker for Early Detection in Dental Caries and Periodontitis. Infect Dis Rep 2020, https://doi.org/10.4081/idr.2020.8722.

60. Cheung, G.Y.C.; Bae, J.S.; Otto, M. Pathogenicity and Virulence of Staphylococcus Aureus. Virulence 2021, 12, 547-569, https://doi.org/10.1080/21505594.2021.1878688.

61. Zhou, X.; Willems, R.J.L.; Friedrich, A.W.; Rossen, J.W.A.; Bathoorn, E. Enterococcus Faecium: From Microbiological Insights to Practical Recommendations for Infection Control and Diagnostics. Antimicrob Resist Infect Control 2020, 9, https://doi.org/10.1186/s13756-020-00770-1.

62. Shahmiri, M.; Mechler, A. The Role of C-Terminal Amidation in the Mechanism of Action of the Antimicrobial Peptide Aurein 1.2. The EuroBiotech Journal 2020, 4, 25-31, https://doi.org/10.2478/ebtj2020-0004.

63. Clark, S.; Jowitt, T.A.; Harris, L.K.; Knight, C.G.; Dobson, C.B. The Lexicon of Antimicrobial Peptides: A Complete Set of Arginine and Tryptophan Sequences. Commun Biol 2021, 4, https://doi.org/10.1038/s42003021-02137-7.

64. Lin, Y.; Malins, L.R. An Electrochemical Approach to Designer Peptide $\alpha$-Amides Inspired by $\alpha$-Amidating Monooxygenase Enzymes. J. Am. Chem. Soc. 2021, 143, 11811-11819, https://doi.org/10.1021/jacs.1c05718.

65. Cytryńska, M.; Zdybicka-Barabas, A. Defense Peptides: Recent Developments. Biomolecular Concepts 2015, 6, 237-251, https://doi.org/10.1515/bmc-2015-0014.

66. Vigerelli, H.; Sciani, J.; Jared, C.; Antoniazzi, M.; Caporale, G.M.; da Silva, A. de C.R.; Pimenta, D.C. Bufotenine Is Able to Block Rabies Virus Infection in BHK-21 Cells. J Venom Anim Toxins incl Trop Dis 2014, 20, https://doi.org/10.1186/1678-9199-20-45.

67. Sarkar, T.; Chetia, M.; Chatterjee, S. Antimicrobial Peptides and Proteins: From Nature's Reservoir to the Laboratory and Beyond. Front. Chem. 2021, 9, https://doi.org/10.3389/fchem.2021.691532.

68. Hazam, P.K.; Phukan, C.; Akhil, R.; Singh, A.; Ramakrishnan, V. Antimicrobial Effects of Syndiotactic Polypeptides. Sci Rep 2021, 11, https://doi.org/10.1038/s41598-021-81394-2.

69. Mangmee, S.; Reamtong, O.; Kalambaheti, T.; Roytrakul, S.; Sonthayanon, P. Antimicrobial Peptide Modifications against Clinically Isolated Antibiotic-Resistant Salmonella. Molecules 2021, 26, https://doi.org/10.3390/molecules26154654. 